\title{
Molecular diagnostics helps to identify distinct subgroups of spinal astrocytomas
}

\author{
Annamaria Biczok ${ }^{1,2^{*}} \mathbb{D}$, Felix L. Strübing ${ }^{3}$, Julia M. Eder ${ }^{3}$, Rupert Egensperger ${ }^{3}$, Oliver Schnell ${ }^{4}$, \\ Stefan Zausinger ${ }^{1,2}$, Julia E. Neumann ${ }^{5}$, Jochen Herms ${ }^{3}$, Joerg-Christian Tonn ${ }^{1,2}$ and Mario M. Dorostkar ${ }^{3}$
}

\begin{abstract}
Primary spinal cord astrocytomas are rare, hence few data exist about the prognostic significance of molecular markers. Here we analyze a panel of molecular alterations in association with the clinical course. Histology and genome sequencing was performed in 26 spinal astrocytomas operated upon between 2000 and 2020. Next-generation DNA/ RNA sequencing (NGS) and methylome analysis were performed to determine molecular alterations. Histology and NGS allowed the distinction of 5 tumor subgroups: glioblastoma IDH wildtype (GBM); diffuse midline glioma H3 K27M mutated (DMG-H3); high-grade astrocytoma with piloid features (HAP); diffuse astrocytoma IDH mutated (DA), diffuse leptomeningeal glioneural tumors (DGLN) and pilocytic astrocytoma (PA). Within all tumor entities GBM (median OS: 5.5 months), DMG-H3 (median OS: 13 months) and HAP (median OS: 8 months) showed a fatal prognosis. DMG-H3 tend to emerge in adolescence whereas GBM and HAP develop in the elderly. HAP are characterized by CDKN2A/B deletion and ATRX mutation. 50\% of PA tumors carried a mutation in the PIK3CA gene which is seemingly associated with better outcome (median OS: PIK3CA mutated 107.5 vs 45.5 months in wildtype PA). This exploratory molecular profiling of spinal cord astrocytomas allows to identify distinct subgroups by combining molecular markers and histomorphology. DMG-H3 tend to develop in adolescence with a similar dismal prognosis like GBM and HAP in the elderly. We here describe spinal HAP with a distinct molecular profile for the first time.
\end{abstract}

Keywords: Spinal astrocytoma, Next-generation sequencing, Prognostic factor, Molecular profile

\section{Introduction}

Since the revision of the WHO classification of central nervous tumors in 2016, molecular alterations are part of the integrated diagnosis of glial tumors [22]. The overwhelming impact of molecular markers has further been revealed as summarized by the latest cIMPACT-NOW update [21-23].

Astrocytic tumors of the spinal cord are rare, especially spinal glioblastoma which account for approximately $1.5 \%$ of all spinal cord tumors $[40,41]$. The majority of spinal cord astrocytomas are located cervicothoracic and

\footnotetext{
*Correspondence: Annamaria.biczok@med.uni-muenchen.de

1 Department of Neurosurgery, Ludwig-Maximilians-University Munich, Marchioninistr. 15, 81377 Munich, Germany
}

Full list of author information is available at the end of the article usually have a short clinical history $[11,15]$. Initial action in the course of treatment consists of surgery to obtain tissue for histological analysis and molecular specification. The value of chemotherapy beyond radiation therapy has yet to be determined as there are no data from dedicated trials available.

Next-generation sequencing (NGS) in astrocytomas in clinical networks is thought to improve diagnostic accuracy, prognostication and discovery of potentially druggable targets.

A Lysine-27-to-methonine (K27M) mutation at one allele of histone $\mathrm{H} 3$ in histologically defined diffuse intrinsic pons gliomas (DIPG), midline gliomas (such as thalamic tumors) and spinal cord astrocytomas has been found to be associated with an aggressive clinical course. This introduced the novel entity 'diffuse midline glioma, original author(s) and the source, provide a link to the Creative Commons licence, and indicate if changes were made. The images or other third party material in this article are included in the article's Creative Commons licence, unless indicated otherwise in a credit line to the material. If material is not included in the article's Creative Commons licence and your intended use is not permitted by statutory regulation or exceeds the permitted use, you will need to obtain permission directly from the copyright holder. To view a copy of this licence, visit http://creativecommons.org/licenses/by/4.0/. The Creative Commons Public Domain Dedication waiver (http://creativeco mmons.org/publicdomain/zero/1.0/) applies to the data made available in this article, unless otherwise stated in a credit line to the data. 
H3 K27-mutand' which is represents approximately $80 \%$ of diffuse intrinsic pontine gliomas (DIPGs), $50 \%$ of thalamic gliomas, and $60 \%$ of spinal cord gliomas [10, 18 , $22,24]$. Recently, it was suggested that the term diffuse midline glioma H3 K27M-mutant should be reserved for tumors that are diffuse (i.e., infiltrating), midline (e.g., thalamus, brainstem, spinal cord, etc.) gliomas with the H3 K27M mutation, and not for other tumors with this mutation, such as ependymomas or midline gangliogliomas [21]. Therefore, treatment decisions are more and more based on molecular profiles of the specific tumor entity, especially if these genetic alterations provide potential targets for therapeutic agents.

To date, there is a lack of information regarding the molecular signature of spinal cord astrocytomas defining potentially prognostic subgroups. To further stratify and characterize the molecular profile of spinal astrocytomas in relation to the clinical outcome we conducted a retrospective study using a next-generation sequencing and methylome analysis.

\section{Materials and methods Study design}

In this retrospective analysis we included patients undergoing surgical resection of primary astrocytoma or glioblastoma of the spinal cord at our institution between 2000 and 2020. Tumors were classified according to the WHO classification of tumors of the central nervous system (2016). We explicitly excluded ependymomas by histological diagnosis, as these have been studied in detail before [44].

Demographic, surgical, and histologic parameters, adjuvant treatment strategies (radio- or chemotherapy use) and neurological outcome were retrieved from the medical records. Surgical treatment was divided into gross total resection (GTR) and subtotal resection (STR) determined on the basis of early postsurgical imaging. The primary endpoint was overall survival (OS).

The cohort was further divided into 5 subgroups, by their unique molecular and histomorphological profile consisting of: glioblastoma multiforme (GBM), diffusemidline gliomas $\mathrm{H} 3$ mutated (DMG-H3), high-grade astrocytoma with piloid features (HAP), diffuse astrocytoma (DA), diffuse leptomeningeal glioneuronal tumor (DLGNT) and pilocytic astrocytoma (PA).

Surgical approach consisted of posterior hemi-laminectomy or laminectomy. Intraoperative neuro-monitoring, ultrasound and microscope were used in all cases. Follow-up was done subsequently in our outpatient department every 3 months with MRI scans.

Tumor progression was defined by either clinical deterioration related to the spinal cord function, new contrast-enhancement or $>25 \%$ volume increase of residual contrast enhancement.

\section{Neuropathological evaluation and microscopy}

Routine neuropathological evaluation of formalin fixed spinal cord glioma samples including hematoxylin and eosin as well as reticulin fiber staining. In addition, immunostaining with antibodies was performed using Ki67 and Olig2.

All histological samples were reviewed by an experienced neuropathologist (MMD) and re-classified based on the 2016 WHO classification of brain tumors [22]. The final diagnoses reported here are based on the combination of histology, genetics and (where available) methylome classifier results including the copy-number profile, which is generated as part of the methylome analysis. IDH-wildtype gliomas lacking necrosis or endothelial proliferation, but with molecular features of glioblastoma are reported here as glioblastoma, IDH wildtype as suggested in the cIMPACT-NOW update 6 [23].

Images were taken on Olympus BX51 microscope, equipped with an Olympus SC30 digital camera, or on a Zeiss Axioscan.Z1, using $20 \times$ objectives. Microscope images shown were digitally adjusted for white balance, exposure, contrast and color saturation.

\section{DNA and RNA isolation}

DNA and, for cases since 2018, RNA, were extracted from micro-dissected FFPE tissue using an automated Maxwell system for DNA (Promega, Fitchburg, Massachusetts, USA) or Allprep DNA/RNA Mini Kit (Quiagen, Hilden, Germany) according to the manufacturer's instructions. Nucleic acid concentrations were determined on a on a Quantus Fluorometer (Promega, Walldorf, Germany) according to the manufacturer's instructions.

\section{DNA methylation profiling}

DNA methylation profiling was performed on cases from which sufficient DNA could be extracted from the tissue. Methylation profiling was done using 100-250 ng DNA on an Illumina Infinium MethylationEPIC BeadChip array (Illumina, San Diego, CA, USA), using the plate reader feature of an Illumina NextSeq 550. The protocols were according to the manufacturer's instructions. Raw methylation data were analyzed using the DNA methylation-based brain tumor classifier (v11b4), which also provides data on copy-number alterations and MGMT promoter methylation [9]. T-stochastic neighborhood embedding ( $t$-SNE) was calculated in R. Presumably because of DNA degradation, many older cases tended not to match definitively (score $>=0.9$ ), but a high score $(>=0.5)$ with compatible histology was deemed sufficient for a diagnosis. The methylation classes and associated 
scores are reported in detail in the Additional file 2: Table S1.

\section{DNA and RNA sequencing}

On initial cases DNA panel sequencing was performed using an Illumina custom amplicon panel which targeted the coding DNA sequence or mutational hotspots of 34 brain tumor associated genes as well as selected regions on chromosomes 1 and 19 for copy-number analysis (Additional file 2: Table S1.). Library preparation was performed according to the manufacturer's instructions using 50-100 ng DNA. Sequencing was performed on an Illumina MiniSeq.

From early 2019 on, we switched to the commercially available Illumina Trusight Oncology 500 (TSO500) panel for all clinical applications, which is a combined DNA and RNA panel. Thus, all recent cases, as well older cases which had become clinically relevant since that time, were sequenced using the TSO500 panel, which covers the coding DNA sequence of 522 cancer-associated genes, the TERT promoter, and the RNA sequence of 55 genes to detect fusions and splice variants. Library preparation was performed according to the manufacturer's instructions using 30-150 ng DNA and 50-85 ng RNA. Sequencing was performed on an Illumina NextSeq 550.

Raw sequencing data were de-multiplexed and aligned to the human genome (GRCh37/hg19) using Illumina software, either on the MiniSeq for the custom panel or a dedicated Unix Server for the TSO500 panel, yielding variant calls, DNA amplifications, as well as fusions and splice variants. Variant calls were further evaluated using custom scripts written in Igor Pro (Wavemetrics, Lake Oswego, OR, USA) which retrieved variant effect prediction data from the ENSEMBL server using a REST API. Variants were subsequently filtered based on variant allele frequency $(\geq 10 \%)$ and minor allele frequency in the population $(\leq 1 \%)$. Variants annotated as "benign" or "likely benign" in ClinVar were rejected. Variants without a ClinVar entry were filtered both on SIFT and PolyPhen predictions, with variants being rejected which had both SIFT "tolerated" and PolyPhen "benign" as entries.

\section{Statistical analysis}

SPSS for Windows (SPSS, Version 26.0, Chicago, IL) was used for statistical calculations. OS and PFS were analyzed with the Kaplan-Meier-method. The distribution of patient- and tumor-related variables was analyzed by chi-squared statistics (for categorical variables) and Kruskal-Wallis-Test (for continuously scaled variables). The median was used as threshold for dichotomization of parameters. For univariate prognostic analyses, all parameters were evaluated using Cox-regression. A twotailed $p$-value $<0.05$ was considered significant.

\section{Results}

\section{Patient characteristics}

In total 26 patients (19 male/7 female) with newly diagnosed astrocytomas were included with sufficient amount and quality of tissue for molecular analyses. The integrated diagnoses, based on histology, sequencing and DNA-methylation patterns were pilocytic astrocytoma ( $\mathrm{n}=8$ patients), glioblastoma, IDH wildtype $(\mathrm{n}=6$ patients), diffuse midline glioma, H3 K27M mutated $(\mathrm{n}=5$ patients), high-grade astrocytoma with piloid features $(\mathrm{n}=4$ patients), diffuse astrocytoma, IDH mutated $(\mathrm{n}=2$ patients $)$ and diffuse leptomeningeal glioneuronal tumor ( $\mathrm{n}=1$ patients). None of these patients were known to have familial tumor predisposition syndromes based on anamnesis. Their clinical characteristics are summarized in Table 1. The length of total follow-up was 19 months.

The median age at diagnosis was 14.9 years for PA, 18.3 years for DMG-H3, 21.5 years for DGLN, 44.3 years for DA, 51.1 years for GBM and 70.3 years for HAP patients, respectively. We correlated the genetic data of all tumors with available clinical parameters. Age varied considerably with a significantly younger patient population in PA, DLGNT and DMG-H3 tumors. The thoracic $(n=13)$ and cervical $(n=13)$ region were affected equally often. When further stratified by tumor grade, patients with higher WHO grade tumors were more likely to receive adjuvant $\mathrm{RT}$ than patients with pilocytic astrocytomas.

\section{Molecular characteristics}

Tumors were classified based on histologic and genetic alterations, as well as the results of methylome analysis, where available. Samples of histological properties are shown in Fig. 1, while Fig. 2 summarizes the overall genomic alterations of all patients, with details listed in the Supplementary Table. Results of t-SNE clustering of cases on which methylome analysis was performed are shown in Fig. 3.

IDH-wildtype glioblastomas were histologically and molecularly heterogeneous. Fusion genes were detected in the two youngest patients: Case \#22, a 1.6 years old boy, had a TPM3-NTRK1 fusion, and case \#26, a 24 years old male, had a FGFR1-PLEC fusion as well as a PDGFRA-FIP1L1 fusion. Both these tumors, however, showed a match to the methylation class "glioblastoma, IDH wildtype, subclass midline" with high calibrated scores (0.99 and 0.97, respectively), confirming the diagnosis of glioblastoma, IDH wildtype. 
Table 1 Patient characteristics and histomorphology

\begin{tabular}{|c|c|c|c|c|c|c|}
\hline & GBM & DMG-H3 & HAP & DA & DLGN & PA \\
\hline Total & 6 & 5 & 4 & 2 & 1 & 8 \\
\hline Age (years) & $51.1(11.6-73.8)$ & $18.3(9.3-53.3)$ & $70.3(44-72.9)$ & $44.3(33.5-55.1)$ & 21.5 & $14.9(2.2-48.9)$ \\
\hline Gender (male/female) & $5 / 1$ & $4 / 2$ & $4 / 0$ & $1 / 0$ & $1 / 1$ & $4 / 3$ \\
\hline $\begin{array}{l}\text { Localization (cervical/tho- } \\
\text { racic/lumbar) }\end{array}$ & $4 / 2 / 0$ & $3 / 2 / 0$ & $1 / 3 / 0$ & $2 / 0 / 0$ & $0 / 2 / 0$ & $3 / 4 / 0$ \\
\hline $\mathrm{EOR}(\mathrm{GTR})$ & 0 & 0 & 0 & 0 & 0 & 2 \\
\hline Adjuvant RT & 5 & 5 & 4 & 2 & 0 & 3 \\
\hline Adjuvant CT & 5 & 4 & 4 & 1 & 0 & 0 \\
\hline Ki67 (median) & 0.25 & 0.2 & 0.1 & 0.02 & 0.3 & 0.1 \\
\hline Necrosis & 1 & 0 & 0 & 0 & 0 & 0 \\
\hline Endothelial Proliferation & 3 & 3 & 0 & 0 & 0 & 0 \\
\hline Mitosis count (median) & 8 & 2.5 & 1 & 0 & 0 & 0 \\
\hline OS (months) & $5.5(1-21)$ & $13(4-33)$ & $8(6-30)$ & $72(8-136)$ & 141 & $80(2-132)$ \\
\hline Death & 3 & 4 & 3 & 1 & 0 & 1 \\
\hline
\end{tabular}

None of the spinal glioblastomas had a TERT promoter mutation, while typical copy-number alterations such as CDKN2A/B deletion or CDK4 amplification were observed in some tumors. Only one single glioblastoma had an ATRX mutation with concomitant loss of nuclear protein expression. Although TP53 mutations are reported in up to $37.5 \%$ of intracranial primary GBM, only one patient carried a TP53 mutations [8]. Interestingly, beside TP53 mutation, \#1 carried multiple mutations (ATRX, DDX3X and KMT2D) and signs of necrosis, hypothesizing a more aggressive phenotype. Three tumors showed methylation of the MGMT promoter region.

Diffuse H3 mutated midline gliomas all harbored a lysine to methionine mutation on codon 28 of the histone gene H3-3A, which is commonly referred to as "K27M" [20]. Additional mutations were heterogeneous, with two tumors each showing frameshift or stop mutations in ATRX and KMT2D. Overall mutational load is higher in DMG-H3 tumors compared to any other malignant tumor subtype (GBM, APP) with an average of 3 mutations per tumor (GBM: 2, HAP: 2.5). Histologically, three tumors showed features of malignancy while the remaining two did not. The latter showed significantly lower number of mutations (median $\mathrm{n}=2$ ) (Spearman-Rho: $\mathrm{r}=0.973, \mathrm{p}=0.005$ ) and were older (median age $=49.3$ years), than their more aggressive counterparts (median $\mathrm{n}=5$ mutations, median age $=15.1$ years)

High-grade astrocytomas with piloid features occurred in elderly patients compared to PA (median age 70.3 yrs vs $14.9 \mathrm{yrs}, p=0.016$ ). Some of these tumors showed endothelial proliferation and appeared histologically as malignant gliomas (Additional file 1: Figure S1.). Most of these tumors showed a homozygous CDKN2A/B deletion. ATRX mutation was confirmed in one patient. MGMT promoter region was methylated in all HAP. Methylation analysis led to a reclassification into a distinct group of these four cases.

IDH mutated astrocytomas were rare, with one IDH1 and one IDH2 mutated tumor in our cohort. While the former had an ATRX mutation and TP53 mutation, like its supratentorial counterparts, the latter lacked further mutations. However, infratentorial IDH mutated astrocytomas were recently described as a separate subgroup, often lacking the typical combination of ATRX and TP53 mutations [3].

Pilocytic astrocytomas showed typical histology, often with prominent Rosenthal fibers and endothelial alterations but low Ki67 labelling indices. Within PA, of all analyzed genes, PIK3CA gene was most frequently altered in $50 \%$ of which E545A being the most common. Tumors with PIK3CA alterations did not differ in age $(p=0.149)$ or localization $(p=0.102)$ compared to PIK3CA wildtype. PIK3CA mutation was associated with longer OS compared to PIK3CA wildtype (median OS: 107.5 vs 45.5 months). One pilocytic astrocytoma which was wildtype in PIK3CA harbored a FGFR1 N546K mutation. Unfortunately, no RNA data was available for any of the pilocytic astrocytomas, so we were not able to assess BRAF or FGFR fusion genes.

One single tumor was classified as diffuse leptomeningeal glioneuronal tumor, with oligodrendroglia-like morphology, loss of ATRX expression, KIAA1549-BRAF fusion and $1 \mathrm{p} / 19 \mathrm{q}$ co-deletion. Clinically, this tumor was characterized by multiple relapses over a course of almost 17 years. 


\section{Treatment}

Two patients harboring a PA received gross total resection and 24 patients underwent subtotal resection. 19 patients received adjuvant radiation therapy immediately following diagnosis (5 GBM, 5 DMG-H3, 4 HAP, 2 DA and 3 PA). Chemotherapy was administered in 5 patients with GBM, 4 patients with DMG-H3, 4 patients with HAP and one patient with DA.

\section{Patient's outcome: univariate}

12/26 patients died: 3/6 GBM, 4/5 DMG-H3 3/4 HAP, $1 / 2 \mathrm{DA}$ and $1 / 8 \mathrm{PA}$ - the DLGNT tumor patient was alive at the time of last follow-up. The clinical prognosis was significantly associated with tumor subtype $(\mathrm{p}=0.002)$, H3 K27M-mutation $(P=0.03)$, male sex $(p=0.05)$ and age $(p=0.002)$, in univariate Cox regression analysis (Table 2). Tumors harboring a mutation in the H3-3Agene presented with a significantly more aggressive clinical course, like GBM IDH wild type or HAP. Survival was shortest in GBM, DMG-H3 or HAP with no significant difference in Kaplan-Meier analyses. ATRX status (mutation versus ATRX wild-type) was not associated with survival, neither was presence or absence of necroses (Table 1). The Kaplan-Meier estimates of OS are shown in Fig. 4. As PIK3CA mutations were found in $50 \%$ of PA, we conducted a subgroup analysis which revealed an association with longer OS in our cohort (median OS: PIK3CA mutated 107.5 vs 45.5 months in wildtype PA). Due to individualized treatment concepts, including microsurgical resection, chemotherapy and radiation no conclusion can currently be drawn as to the effectiveness of particular therapeutic strategies.

\section{Discussion}

Since the revision of the WHO classification of central nervous system tumors in 2016 tumors are classified by an integrated diagnosis combining histologic and molecular features [22]. Astrocytomas of the spinal cord are infrequent and up-to-date knowledge of their respective tumor biology and molecular alterations is sparse. Thus, a consensus on the treatment of spinal cord astrocytomas according to the biology of these tumors has yet to be determined [11]. Due to the infiltrating nature of these tumors safe gross total resection is rarely feasible, especially within the high-grade counterparts [16, 25]. Current treatment strategies for diffuse midline gliomas, as recommended by the EANO guidelines, consist of radiotherapy alone or TMZ plus radiotherapy followed by TMZ as first line therapy [37]. Our study focused on the clinical, histological and molecular characterization of spinal cord gliomas. To gain more insight into potentially clinically relevant molecular alterations we additionally analyzed a large set of molecular markers in all tumors.

Mutations in genes affecting the histone code and alteration of histone methylation status have been detected in several cancer entities. Interestingly, mutations within a histone gene were initially discovered in gliomas [19, 39]. Mutation in the $\mathrm{K} 27 \mathrm{M}$ position of the histone $\mathrm{H} 3-3 \mathrm{~A}$ gene leads to a block of methylation on the histone $\mathrm{H} 3$ tail inhibiting the cascade responsible for glial differentiation [12]. Other entities such as ependymomas, subependymomas and glioneuronal tumors may rarely harbor H3K27M mutations, however their prognostic value in these tumors is less clear [27, 28, 32, 42].

We found 5/26 (19.2\%) H3-3A K27M positive cases. Ishi et al. found $18.9 \%$ of H3-3A K27M mutations in adult patients while $\mathrm{Yi}$ et al. reported a frequency of $80 \%(20$ out of 25 patients) in a similar sized collective compared to our study $[17,43]$.

In accordance with previous reports the H3-3A $\mathrm{K} 27 \mathrm{M}$ mutation is associated with a fatal prognosis mimicking the clinical course of grade IV tumors like glioblastoma multiforme WHO grade IV. However, most of the published studies included intracranial tumor manifestations resulting in a significant underrepresentation of spinal cord localization. The mutated tumors were associated with worse prognosis in comparison to H3-3A wild-type tumors regardless of tumor grade or location $[6,7,13]$. Our study confirmed these results for DMG-H3 spinal gliomas without any prognostic distinction regarding proliferation or histological grading within this subgroup. However, our sample size of H3-3A K27M mutated tumors did not allow further statistical analysis. Chai et al. and others found a longer survival for H3-3A K27M-mutanted gliomas with less anaplastic features compared to H3-3A K27M-mutant WHO grade IV or H3-3A K27M-wild-type WHO grade IV gliomas, suggesting a prognostic distinction of histomorphological appearance $[10,45]$. In contrast, Yi et al. reported a more favorable prognosis of H3-3A K27M mutant tumors compared to H3-3A K27M-wild-type

(See figure on next page.)

Fig. 1 Histological features of spinal astrocytomas, hematoxylin and eosin stainings (H\&E; left column) and Ki67 immunohistochemistry (right column). PA: pilocytic astrocytoma; GBM: glioblastoma IDH-wild-type; DMG-H3: diffuse midline glioma, H3 K27M mutated; HAP: high-grade astrocytoma with piloid features; Astro, IDH: diffuse astrocytoma IDH 1/2 mutated; DLGNT: diffuse leptomeningeal glioneuronal tumor Scale bar, $100 \mu \mathrm{m}$ 

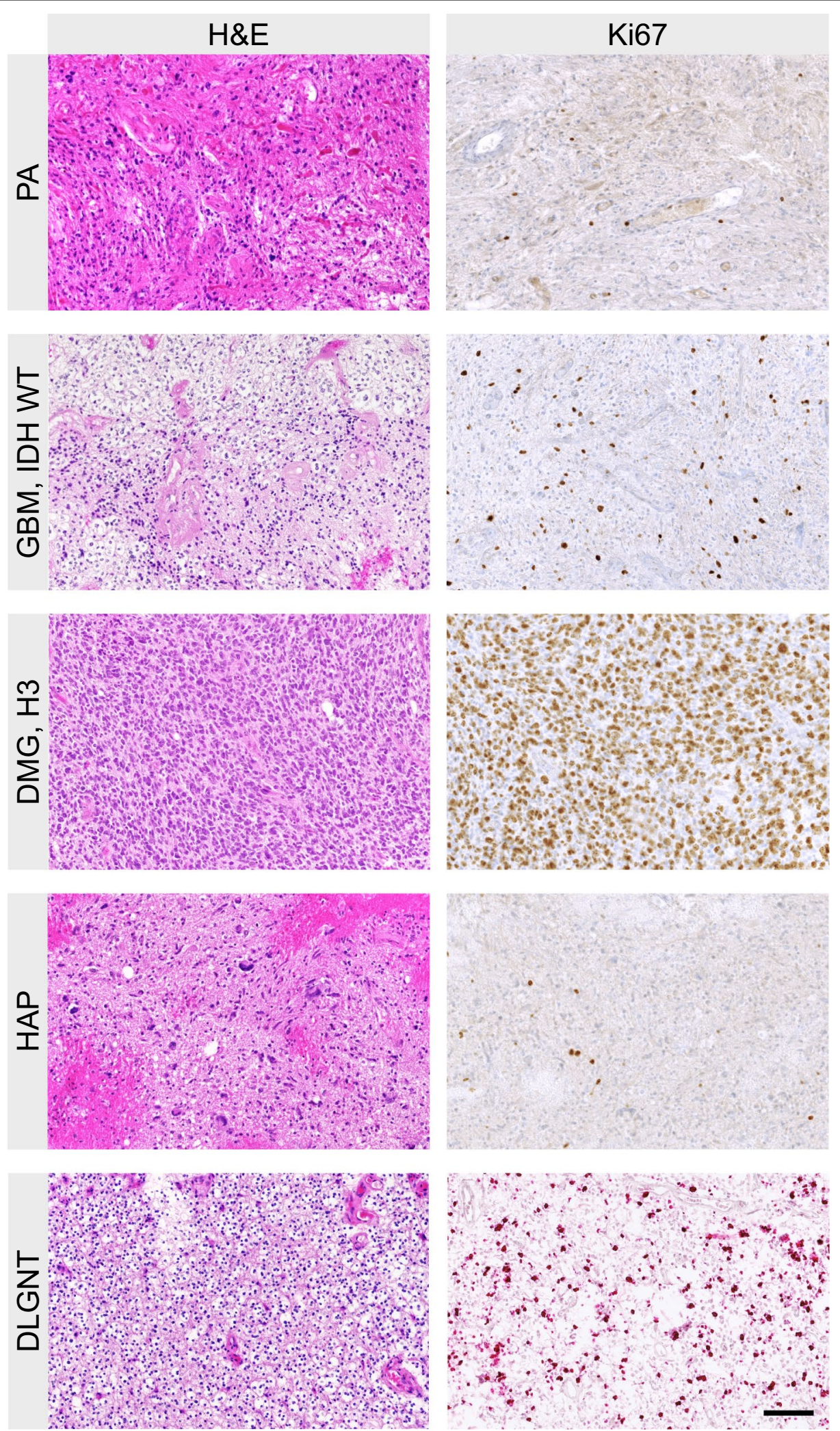

Fig. 1 (See legend on previous page.) 


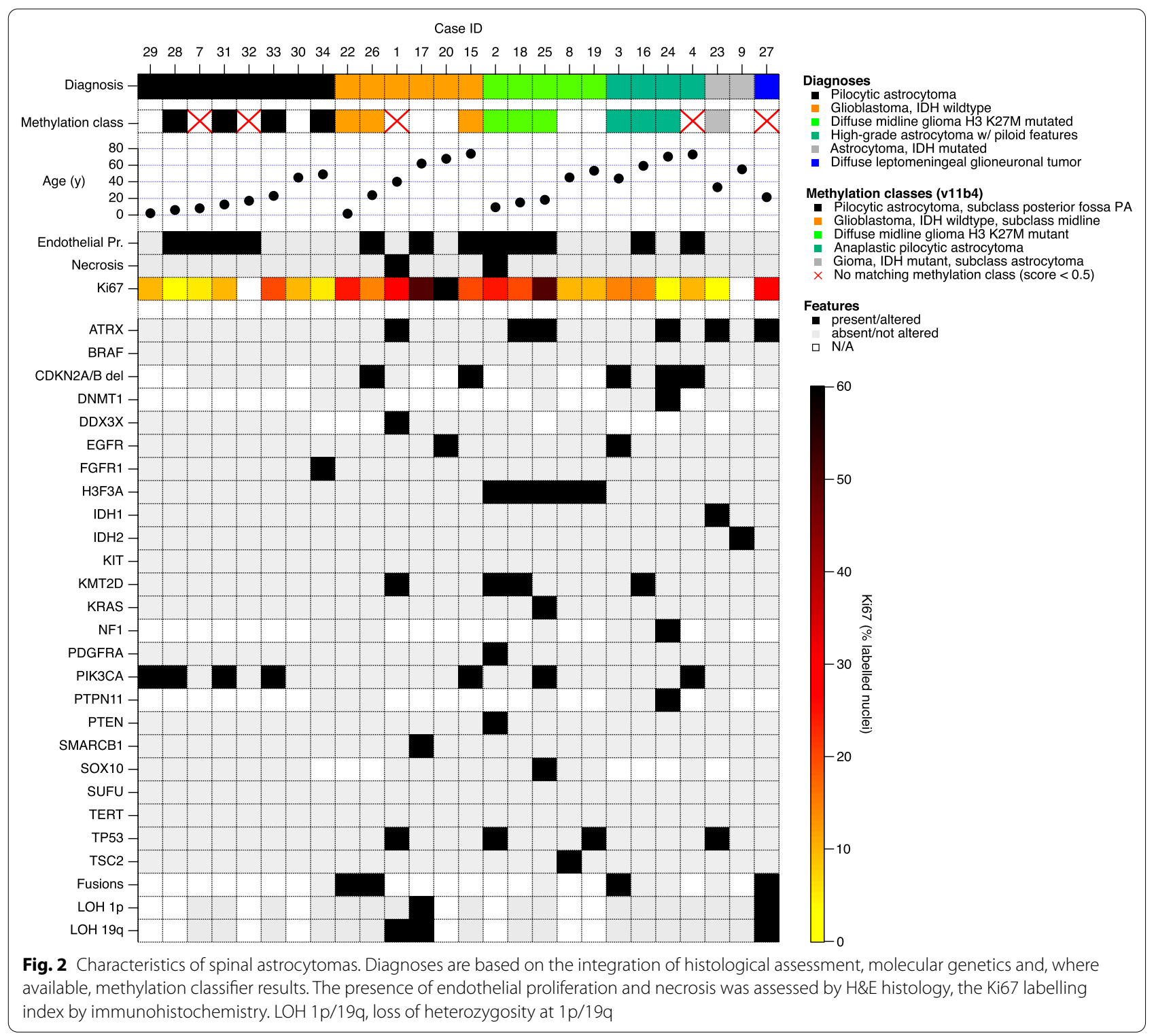

without addressing the histomorphological appearance within the H3-3A K27M-mutant cohort [43].

IDH-wildtype glioblastoma in our cohort were heterogeneous, both clinically and molecularly, suggesting that several distinct molecular entities are currently subsumed in this diagnosis. For instance, the tumor of youngest patient (case \#22) had a NTRK1 fusion gene, which is typical for a subgroup of infant hemispheric gliomas with receptor-tyrosine kinase activation, yet the current methylome classifier (v11b4) produced a high calibrated score (0.99) for the methylation class "glioblastoma, IDH wildtype, subclass midline" [14]. Similarly, case \#26, a malignant glioma with FGFR1 fusion and deletions of CDKN2A/B and TP53 in a young patient, matched to this group with a calibrated score of 0.97 . As a match to a glioblastoma methylation class is considered to be a molecular feature of glioblastoma and due to the current lack of a better suited tumor class, we classified these tumors as glioblastoma [5]. Nevertheless, their respective clinical and molecular features may warrant different diagnoses in the future.

None of the glioblastomas in our cohort had a TERT promoter mutation, which is common in the supratentorial counterparts. However, another recent study on spinal cord gliomas described three cases harboring TERT promoter mutations, suggesting that these do occur in spinal glioblastoma, though probably at a lower frequency than in non-spinal glioblastoma [2]. 
In our study patients with either DMG-H3 (median OS: 13 months), GBM (median OS: 5.5 months) or HAP (median OS: 8 months) had worse survival rates compared to DA (median OS: 72 months) or PA (median OS: 141 months). Patients with DMG-H3 were predominantly younger in accordance with previous reports [1, $26,34]$. Thus, we assume an analogy of the grim prognosis in spinal wild-type GBM in elderly and DMG-H3 in young age. In a study by Perwein et al. dealing with pediatric spinal astrocytomas no patient showed a K27M alteration of the H3-3A gene, however this result was solely based on immunohistochemistry [29]. Interestingly, we found high proliferating activity within DMG$\mathrm{H} 3$ tumors to be associated with a high mutational burden. High mutational burden has been described to be linked with worse outcome in supratentorial gliomas [36]. Larger cohorts have to be analyzed to address this question in spinal gliomas as well.

Within the DA cohort, which had a longer median OS compared to HAP, DMG-H3 and GBM, we found two tumors with a IDH 1 or 2 mutation $(\mathrm{n}=1$ each). Since only one similar case has been reported up to now, the prognostic value of IDH $1 / 2$ mutations has yet to be determined in larger, potentially collaborative studies [35].

HAP have been defined as a distinct, molecular entity recently [30], with only few cases reported in the spinal cord [4]. A definitive diagnosis of these tumors currently relies on methylation analysis [23]. We found several spinal HAP, which had an unfavorable clinical course with a short median OS of 8 months, which is considerably shorter than reported previously for tumors primarily stemming from locations outside the spinal cord $[4,30]$.

For the first time we here present HAP being located in the spinal cord, these patients had a short median OS of 8 months. These tumors with histomorphological characteristics of PA, but with increased mitotic index and other high-grade features have so far been described for intracranial gliomas (Additional file 1: Figure S1.). They harbor alterations in the MAPK pathway as well as alterations of CDKN2A/B and ATRX and display a fatal clinical course [30]. In line with other studies, we also found

\section{(See figure on next page.)}

Fig. 3 t-SNE clustering of analyzed cases against the DKFZ reference dataset [9]. Reference cases are colored according to the respective methylation classes. A: pilocytic astrocytoma; GBM: glioblastoma IDH-wild-type; DMG-H3: diffuse midline glioma, H3 K27M mutated; HAP: high-grade astrocytoma with piloid features; Astro, IDH: diffuse astrocytoma IDH 1/2 mutated; DLGNT: diffuse leptomeningeal glioneuronal tumor. Abbreviations of reference classes: A, IDH: astrocytoma, IDH mutant; A, IDH HG: astrocytoma, IDH mutant, high-grade; ANA, PA: high-grade astrocytoma with piloid features; ATRT, MYC: atypical teratoid/rhabdoid tumor, MYC-associated; ATRT, SHH: atypical teratoid/rhabdoid tumor, sonic hedgehog/Notch-associated; ATRT, TYR: atypical teratoid/rhabdoid tumor, tyrosinase-associated; CHGL: chordoid glioma; CHORDM: chordoma; CN: central neurocytoma; CNS, NB FOXR2: CNS neurolastoma, FOXR2-activated; CONTR, ADENOPIT: control tissue, adenohypophysis; CONTR, CEBM: control tissue, cerebellar hemisphere; CONTR, HEMI: control tissue, cerebral hemispheric cortex; CONTR, HYPTHAL: control tissue, hypothalamus; CONTR, INFLAM: control tissue, inflammatory tumor microenvironment; CONTR, PINEAL: control tissue, pineal gland; CONTR, PONS: control tissue, pons; CONTR, REACT: control tissue, reactive tumor microenvironment; CONTR, WM: control tissue, white matter; $C P H$, ADM: adamantinomatous craniopharyngeoma; CPH, PAP: papillary craniopharyngeoma; DLGNT: diffuse leptomeningeal glioneuronal tumor; DMG, K27: diffuse midline glioma, H3 K27M mutant; EFT, CIC: CNS Ewing Sarcoma Family Tumor with CIC alteration; ENB, A: esthesioneuroblastoma, subclass A; ENB, B: esthesioneuroblastoma, subclass B; EPN, MPE: myxopapillary ependymoma; EPN, PF A: ependymoma, posterior fossa A; EPN, PF B: ependymoma, posterior fossa B; EPN, RELA: ependymoma, RELA fusion positive; EPN, SPINE: ependymoma, spinal; EPN, YAP: ependymoma, YAP fusion positive; ETMR: embryonal tumor with multilayered rosettes; EWS: Ewing sarcoma; GBM, G34: glioblastoma, IDH wildt-type, H3-3 G34 mutant; GBM, MES: glioblastoma, IDH wild-type, mesenchymal; GBM, MID: glioblastoma, IDH wild-type, midline; GBM, MYCN: glioblastoma, IDH wild-type, MYCN-associated; GBM, RTK I: glioblastoma, IDH wild-type, receptor tyrosine kinase I; GBM, RTK II: glioblastoma, IDH wild-type, receptor tyrosine kinase II; GBM, RTK III: glioblastoma, IDH wild-type, receptor tyrosine kinase III; HGNET, BCOR: CNS high grade neuroepithelial tumor with BCOR alteration; HGNET, MN1: CNS high grade neuroepithelial tumor with MN1 alteration; HMB: hemangioblastoma; IHG: infantile hemispheric glioma; LGG, DNT: low-grade glioma, dysembryoplastic neuroepithelial tumor; LGG, GG: low-grade glioma, ganglioglioma; LGG, MYB: low-grade glioma, MYB/MYBL1 altered; LGG, PA MID: low-grade glioma, midline pilocytic astrocytoma; LGG, PA PF: low-grade glioma, posterior fossa pilocytic astrocytoma; LGG, PA/GG ST: low-grade glioma, hemispheric pilocytic astrocytoma and ganglioglioma; LGG, RGNT: low-grade glioma, rosette-forming glioneuronal tumor; LGG, SEGA: low-grade glioma, subependymal giant-cell astrocytoma; LIPN: cerebellar liponeurocytoma; LYMPHO: lymphoma; MB, G3: medulloblastoma, group 3; MB, G4: medulloblastoma, group 4; MB, SHH CHL ADL: medulloblastoma, sonic hedgehog activated A (children/adult); MB, SHH INF: medulloblastoma, sonic hedgehog activated B (infant); MB, WNT: medulloblastoma, WNT activated; MELAN: malignant melanoma; MELCYT: melanocytoma; MNG: meningioma; O, IDH: oligodendroglioma, IDH-mutant; PGG, nC: paraganglioma, spinal non-CIMP; PIN, T PB A: pineoblastoma group A / intracranial retinoblastoma; PIN, T PB B: pineoblastoma group B; PIN, T PPT: pineal parenchymal tumor; PITAD, ACTH: pituitary adenoma, ACTH; PITAD, FSH LH: pituitary adenoma, FSH/LH; PITAD, PRL: pituitary adenoma, prolactin; PITAD, STH DNS A: pituitary adenoma, STH densely granulated, group A; PITAD, STH DNS B: pituitary adenoma, STH densely granulated, group B; PITAD, STH SPA: pituitary adenoma, STH sparsely granulated; PITAD, TSH: pituitary adenoma, TSH; PITUI: pituicytoma / granular cell tumor / spindle cell oncocytoma; PLASMA: plasmacytoma; PLEX, AD: plexus tumor, subclass adult; PLEX, PED A: plexus tumor, subclass pediatric A; PLEX, PED B: plexus tumor, subclass pediatric B; PTPR, A: papillary tumor of the pineal region, group A; PTPR, B: papillary tumor of the pineal region, group B; PXA: pleomorphic xanthoastrocytoma; RETB: retinoblastoma; SCHW: schwannoma; SCHW, MEL: melanotic schwannoma; SFT, HMPC: solitary fibrous tumor / hemangiopericytoma; SUBEPN, PF: subependymoma, posterior fossa; SUBEPN, SPINE: subependymoma, spinal; SUBEPN, ST: subependymoma, supratentorial; 


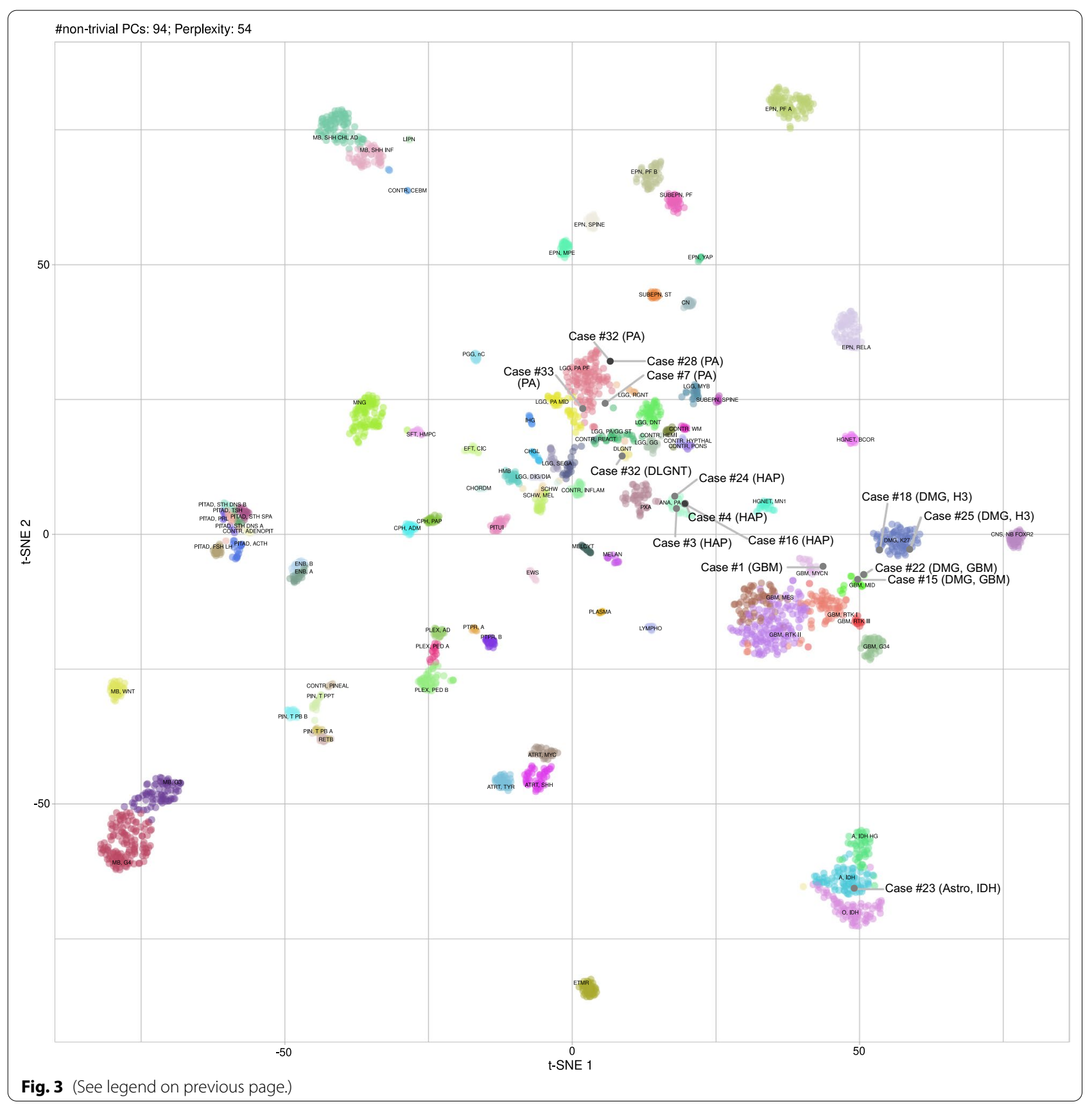

a higher median age of patients with HAP compared to PA [30, 31]. We were also able to confirm in HAP alterations in CDKN2A/B in three tumors and an ATRX alteration in one specimen. The MGMT promoter region was methylated in all cases, thus significantly higher in comparison to their intracranial counterparts (45\%) [30].

In PA, we could detect a mutation of the PIK3CA gene in $50 \%$ of our patients, which are rarely reported in pilocytic astrocytomas outside the spinal cord. Among low-grade CNS tumors, PIK3CA mutations have been described in rosette-forming glioneuronal tumors although they also occur in glioblastoma [33, 38]. Interestingly, in our study PIK3CA mutation seems to be associated with longer OS, which may be caused by its association with pilocytic astrocytoma, which in turn had the most favorable overall outcome. This preliminary signal derived from a small cohort needs to be validated in a larger dataset.

This study is limited by its retrospective study design and the heterogeneity of postsurgical treatment regimes. 
Table 2 Univariate analysis

\begin{tabular}{|c|c|c|c|}
\hline & \multirow{2}{*}{$\begin{array}{l}\text { Univariate analysis } \\
p \text {-value }\end{array}$} & \multirow[b]{2}{*}{$H R$} & \multirow[b]{2}{*}{$95 \% \mathrm{Cl}$ for $\mathrm{HR}$} \\
\hline & & & \\
\hline Age (continuously) & 0.002 & 1.063 & $1.022-1.105$ \\
\hline Female Gender (vs. male) & 0.05 & 0.127 & $0.016-0.998$ \\
\hline Localization (cervical vs. thoracic) & 0.228 & 0.509 & $0.170-1.526$ \\
\hline Mutation H3F3A (yes vs.no) & 0.03 & 4.164 & $1.038-15.801$ \\
\hline ATRX loss (yes vs. no) & 0.085 & 0.119 & $0.011-1.337$ \\
\hline Necrosis (yes vs. no) & 0.650 & 0.045 & $0.136-9.092$ \\
\hline Endothelial Proliferation (yes vs. no) & 0.222 & 2.108 & $0.637-6.977$ \\
\hline Mitosis (continuously) & 0.059 & 1.653 & $0.981-2.784$ \\
\hline Ki-67 (continuously) & 0.057 & 10.428 & $0.75-43.398$ \\
\hline Tumor Subtype & 0.002 & 0.42 & $0.244-0.724$ \\
\hline Adjuvant Treatment (yes vs no) & 0.154 & 3.063 & $0.657-14.289$ \\
\hline
\end{tabular}

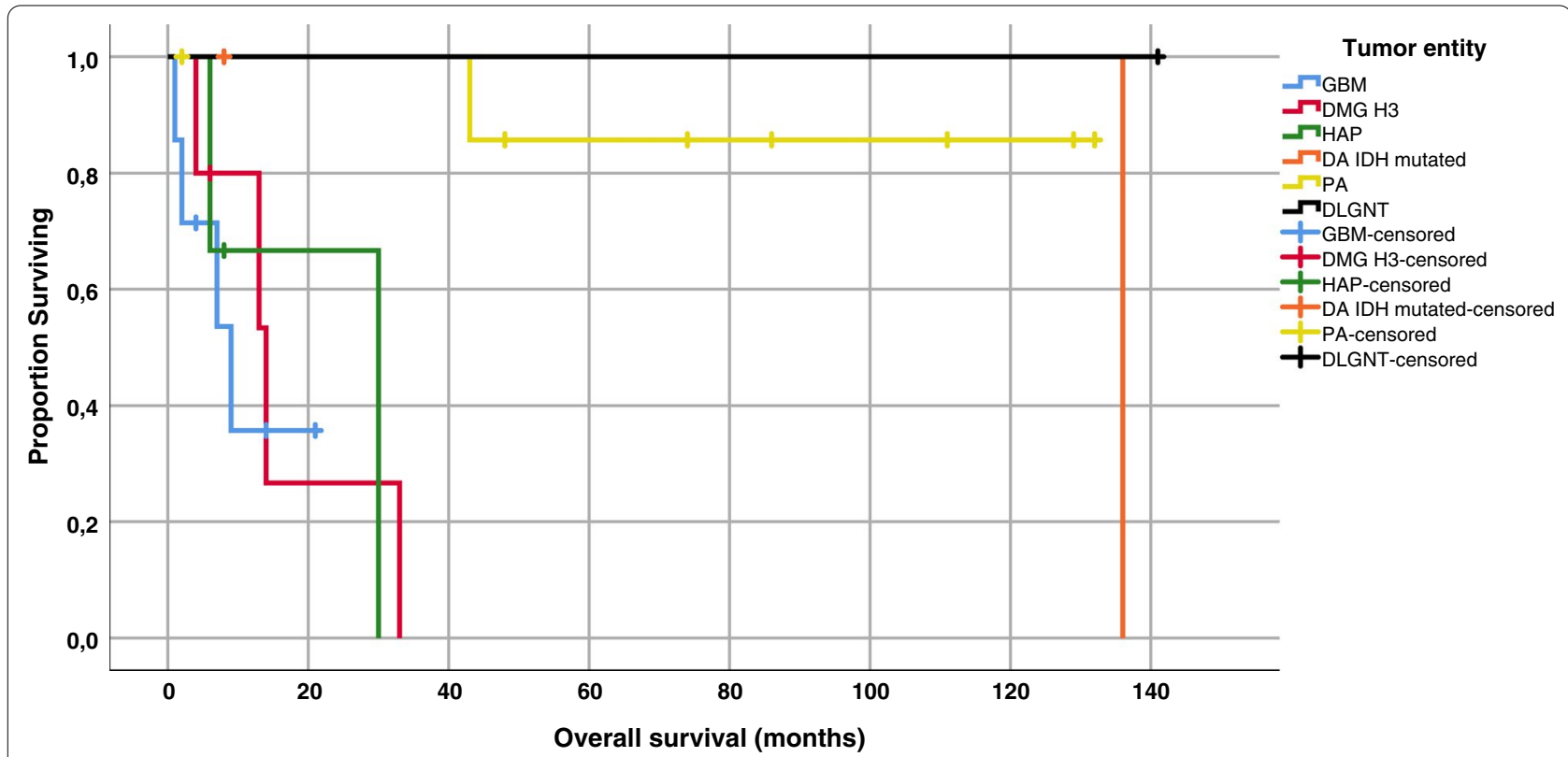

Fig. 4 Kaplan-Meier analysis of overall survival stratified by subgroup (GBM, DMG-H3, HAP, DA, DLGNT and PA)

Moreover, comparison between subgroups is hampered by small patient numbers. Nevertheless, the analysis of larger panels of molecular markers might provide additional knowledge of tumor biology and clinical behavior in spinal gliomas as has been found for the cerebral counterparts in the past.

\section{Conclusion}

Spinal astrocytomas are comprised of histomorphological heterogeneous subgroups of tumors with distinct molecular alterations. DMG-H3 tumors with H3-3A mutations tend to develop in adolescence and have a similar dismal prognosis like GBM and HAP in the elderly. HAP within the spinal cord, harboring a specific molecular signature, is described here for the first time, having a similar dismal prognosis as their supratentorial counterparts. PA with a mutated PIK3CA gene seemingly have longer OS.

Analysis of molecular alterations in spinal astrocytomas might be of added value for diagnostic accuracy and clinical management. Future collaborative clinical trials in larger cohorts are needed. 


\section{Supplementary Information}

The online version contains supplementary material available at https://doi. org/10.1186/s40478-021-01222-6.

Additional file 1. Characteristics of spinal high-grade astrocytomas with piloid features. Micropgraphs of H\&E-stained sections of cases \#3, \#4, \#16 and \#23 are shown alongside Manhattan plots showing their respective copy-number profiles. Cases \#3, \#4, and \#23 had deletions of the CDKN2A/B genes, highlighted by blue arrows. Scale bar, $100 \mu \mathrm{m}$

Additional file 2. Detailed results of histological assessment and molecular analysis.

\section{Authors' contributions}

All authors have contributed to the manuscript. A.B.: development of study concept, composition of manuscript, statistical analysis. F.L.S.: NGS analysis. J.M.E.: methylome analysis. R.E.: review of histologic specimen. O.S.: manuscript editing. S.Z.: manuscript editing. J.E.N.: methylome analysis. J.H.: manuscript editing. J.C.T: composition of manuscript. M.M.D.: development of study concept, composition of manuscript, NGS analysis.

\section{Funding}

Open Access funding enabled and organized by Projekt DEAL. None.

\section{Availability of data and materials}

The dataset supporting the conclusions of this article is included within the article as supplementary material. Other data is available from the corresponding author on reasonable request.

\section{Declarations}

\section{Consent for publication}

Not applicable.

\section{Ethic approval}

Tissue sample and data collection were performed in accordance and approval by the local ethics committee. Institutional ethic board approval was obtained (approval number: 18-747).

\section{Competing interest}

The authors declare that they have no competing interest.

\section{Author details}

'Department of Neurosurgery, Ludwig-Maximilians-University Munich, Marchioninistr. 15, 81377 Munich, Germany. ${ }^{2}$ Partner Site Munich, and German Cancer Research Center (DKFZ), German Cancer Consortium (DKTK), Heidelberg, Germany. ${ }^{3}$ Center for Neuropathology and Prion Research, Ludwig-Maximilians-University Munich, Munich, Germany. ${ }^{4}$ Department of Neurosurgery, Medical Center - University of Freiburg, Freiburg, Germany. ${ }^{5}$ Institute of Neuropathology, University Medical Center Hamburg-Eppendorf, Hamburg, Germany.

Received: 22 May 2021 Accepted: 19 June 2021

Published online: 30 June 2021

\section{References}

1. Aihara K, Mukasa A, Gotoh K, Saito K, Nagae G, Tsuji S, Tatsuno K, Yamamoto S, Takayanagi S, Narita Y et al (2014) H3F3A K27M mutations in thalamic gliomas from young adult patients. Neuro Oncol 16:140-146. https://doi.org/10.1093/neuonc/not144

2. Alvi MA, Ida CM, Paolini MA, Kerezoudis P, Meyer J, Barr Fritcher EG, Goncalves S, Meyer FB, Bydon M, Raghunathan A (2019) Spinal cord high-grade infiltrating gliomas in adults: clinico-pathological and molecular evaluation. Mod Pathol 32:1236-1243. https://doi.org/10.1038/ s41379-019-0271-3
3. Banan R, Stichel D, Bleck A, Hong B, Lehmann U, Suwala A, Reinhardt A, Schrimpf D, Buslei R, Stadelmann C et al (2020) Infratentorial IDH-mutant astrocytoma is a distinct subtype. Acta Neuropathol 140:569-581. https:// doi.org/10.1007/s00401-020-02194-y

4. Bender K, Perez E, Chirica M, Onken J, Kahn J, Brenner W, Ehret F, Euskirchen P, Koch A, Capper D et al (2021) High-grade astrocytoma with piloid features (HGAP): the Charite experience with a new central nervous system tumor entity. J Neurooncol 153:109-120. https://doi.org/10.1007/ s11060-021-03749-z

5. Brat DJ, Aldape K, Colman H, Holland EC, Louis DN, Jenkins RB, Kleinschmidt-DeMasters BK, Perry A, Reifenberger G, Stupp R et al (2018) CIMPACT-NOW update 3: recommended diagnostic criteria for "Diffuse astrocytic glioma, IDH-wildtype, with molecular features of glioblastoma, WHO grade IV." Acta Neuropathol 136:805-810. https://doi.org/10.1007/ s00401-018-1913-0

6. Buczkowicz P, Bartels U, Bouffet E, Becher O, Hawkins C (2014) Histopathological spectrum of paediatric diffuse intrinsic pontine glioma: diagnostic and therapeutic implications. Acta Neuropathol 128:573-581. https://doi.org/10.1007/s00401-014-1319-6

7. Buczkowicz P, Hawkins C (2015) Pathology, Molecular Genetics, and Epigenetics of Diffuse Intrinsic Pontine Glioma. Front Oncol 5:147. https:// doi.org/10.3389/fonc.2015.00147

8. Cancer Genome Atlas Research N (2008) Comprehensive genomic characterization defines human glioblastoma genes and core pathways. Nature 455:1061-1068. https://doi.org/10.1038/nature07385

9. Capper D, Jones DTW, Sill M, Hovestadt V, Schrimpf D, Sturm D, Koelsche C, Sahm F, Chavez L, Reuss DE et al (2018) DNA methylation-based classification of central nervous system tumours. Nature 555:469-474. https:// doi.org/10.1038/nature26000

10. Chai RC, Zhang YW, Liu YQ, Chang YZ, Pang B, Jiang T, Jia WQ, Wang YZ (2020) The molecular characteristics of spinal cord gliomas with or without H3 K27M mutation. Acta Neuropathol Commun 8:40. https://doi. org/10.1186/s40478-020-00913-w

11. Chaichana KL, Jallo GI, McGirt MJ, Goldstein IM, Tobias ME, Kothbauer KF (2008) Extent of surgical resection of malignant astrocytomas of the spinal cord: outcome analysis of 35 patients. Neurosurgery 63:55-61. https://doi.org/10.1227/01.Neu.0000335070.37943.09

12. Chan KM, Fang D, Gan H, Hashizume R, Yu C, Schroeder M, Gupta N, Mueller S, James CD, Jenkins R et al (2013) The histone H3.3K27M mutation in pediatric glioma reprograms H3K27 methylation and gene expression. Genes Dev 27:985-990. https://doi.org/10.1101/gad.217778.113

13. Feng J, Hao S, Pan C, Wang Y, Wu Z, Zhang J, Yan H, Zhang L, Wan H (2015) The H3.3 K27M mutation results in a poorer prognosis in brainstem gliomas than thalamic gliomas in adults. Hum Pathol 46:1626-1632. https:// doi.org/10.1016/j.humpath.2015.07.002

14. Guerreiro Stucklin AS, Ryall S, Fukuoka K, Zapotocky M, Lassaletta A, Li C, Bridge T, Kim B, Arnoldo A, Kowalski PE et al (2019) Alterations in ALK ROS1/NTRK/MET drive a group of infantile hemispheric gliomas. Nat Commun 10:4343. https://doi.org/10.1038/s41467-019-12187-5

15. Guidetti B, Mercuri S, Vagnozzi R (1981) Long-term results of the surgical treatment of 129 intramedullary spinal gliomas. J Neurosurg 54:323-330. https://doi.org/10.3171/jns.1981.54.3.0323

16. Houten JK, Cooper PR (2000) Spinal cord astrocytomas: presentation, management and outcome. J Neurooncol 47:219-224. https://doi.org/ 10.1023/a:1006466422143

17. Ishi Y, Takamiya S, Seki T, Yamazaki K, Hida K, Hatanaka KC, Ishida Y, Oda Y, Tanaka S, Yamaquchi S (2020) Prognostic role of H3K27M mutation, histone H3K27 methylation status, and EZH2 expression in diffuse spinal cord gliomas. Brain Tumor Pathol 37:81-88. https://doi.org/10.1007/ s10014-020-00369-9

18. Karremann M, Gielen GH, Hoffmann M, Wiese M, Colditz N, WarmuthMetz M, Bison B, Claviez A, van Vuurden DG, von Bueren AO et al (2018) Diffuse high-grade gliomas with $\mathrm{H} 3 \mathrm{~K} 27 \mathrm{M}$ mutations carry a dismal prognosis independent of tumor location. Neuro Oncol 20:123-131. https:// doi.org/10.1093/neuonc/nox149

19. Khuong-Quang DA, Buczkowicz P, Rakopoulos P, Liu XY, Fontebasso AM, Bouffet E, Bartels U, Albrecht S, Schwartzentruber J, Letourneau L et al (2012) K27M mutation in histone H3.3 defines clinically and biologically distinct subgroups of pediatric diffuse intrinsic pontine gliomas. Acta Neuropathol 124:439-447. https://doi.org/10.1007/s00401-012-0998-0 
20. Leske H, Rushing E, Budka H, Niehusmann P, Pahnke J, Panagopoulos I (2018) K27/G34 versus K28/G35 in histone H3-mutant gliomas: A note of caution. Acta Neuropathol 136:175-176. https://doi.org/10.1007/ s00401-018-1867-2

21. Louis DN, Giannini C, Capper D, Paulus W, Figarella-Branger D, Lopes MB, Batchelor TT, Cairncross JG, van den Bent M, Wick W et al (2018) CIMPACTNOW update 2: diagnostic clarifications for diffuse midline glioma, H3 K27M-mutant and diffuse astrocytoma/anaplastic astrocytoma, IDH-mutant. Acta Neuropathol 135:639-642. https://doi.org/10.1007/ s00401-018-1826-y

22. Louis DN, Perry A, Reifenberger G, von Deimling A, Figarella-Branger D, Cavenee WK, Ohgaki H, Wiestler OD, Kleihues P, Ellison DW (2016) The 2016 World Health Organization Classification of Tumors of the Central Nervous System: a summary. Acta Neuropathol 131:803-820. https://doi. org/10.1007/s00401-016-1545-1

23. Louis DN, Wesseling P, Aldape K, Brat DJ, Capper D, Cree IA, Eberhart C, Figarella-Branger D, Fouladi M, Fuller GN et al (2020) CIMPACT-NOW update 6: new entity and diagnostic principle recommendations of the CIMPACT-Utrecht meeting on future CNS tumor classification and grading. Brain Pathol 30:844-856. https://doi.org/10.1111/bpa.12832

24. Maeda S, Ohka F, Okuno Y, Aoki K, Motomura K, Takeuchi K, Kusakari $\mathrm{H}$, Yanagisawa N, Sato S, Yamaguchi J et al (2020) H3F3A mutant allele specific imbalance in an aggressive subtype of diffuse midline glioma, $\mathrm{H3}$ K27M-mutant. Acta Neuropathol Commun 8:8. https://doi.org/10.1186/ s40478-020-0882-4

25. McGirt MJ, Goldstein IM, Chaichana KL, Tobias ME, Kothbauer KF, Jallo GI (2008) Extent of surgical resection of malignant astrocytomas of the spinal cord: outcome analysis of 35 patients. Neurosurgery 63: 55-60; discussion 60-51 https://doi.org/10.1227/01.NEU.0000335070.37943.09

26. Meyronet D, Esteban-Mader M, Bonnet C, Joly MO, Uro-Coste E, AmielBenouaich A, Forest F, Rousselot-Denis C, Burel-Vandenbos F, Bourg V et al (2017) Characteristics of H3 K27M-mutant gliomas in adults. Neuro Oncol 19:1127-1134. https://doi.org/10.1093/neuonc/now274

27. Nguyen AT, Colin C, Nanni-Metellus I, Padovani L, Maurage CA, Varlet P, Miquel C, Uro-Coste E, Godfraind C, Lechapt-Zalcman E et al (2015) Evidence for BRAF V600E and H3F3A K27M double mutations in paediatric glial and glioneuronal tumours. Neuropathol Appl Neurobiol 41:403-408. https://doi.org/10.1111/nan.12196

28. Pages M, Beccaria K, Boddaert N, Saffroy R, Besnard A, Castel D, Fina F, Barets D, Barret E, Lacroix L et al (2018) Co-occurrence of histone H3 K27M and BRAF V600E mutations in paediatric midline grade I ganglioglioma. Brain Pathol 28:103-111. https://doi.org/10.1111/bpa.12473

29. Perwein T, Benesch M, Kandels D, Pietsch T, Schmidt R, Quehenberger F, Bison B, Warmuth-Metz M, Timmermann B, Krauss J, et al (2020) High frequency of disease progression in pediatric spinal cord low-grade glioma (LGG): Management strategies and results from the German LGG study group. Neuro Oncol, https://doi.org/10.1093/neuonc/noaa296

30. Reinhardt A, Stichel D, Schrimpf D, Sahm F, Korshunov A, Reuss DE, Koelsche C, Huang K, Wefers AK, Hovestadt V et al (2018) Anaplastic astrocytoma with piloid features, a novel molecular class of IDH wildtype glioma with recurrent MAPK pathway, CDKN2A/B and ATRX alterations. Acta Neuropathol 136:273-291. https://doi.org/10.1007/s00401-018-1837-8

31. Rodriguez FJ, Scheithauer BW, Burger PC, Jenkins S, Giannini C (2010) Anaplasia in pilocytic astrocytoma predicts aggressive behavior. Am J Surg Pathol 34:147-160. https://doi.org/10.1097/PAS.0b013e3181c75238

32. Ryall S, Guzman M, Elbabaa SK, Luu B, Mack SC, Zapotocky M, Taylor MD, Hawkins C, Ramaswamy V (2017) H3 K27M mutations are extremely rare in posterior fossa group A ependymoma. Childs Nerv Syst 33:1047-1051. https://doi.org/10.1007/s00381-017-3481-3

33. Sievers P, Appay R, Schrimpf D, Stichel D, Reuss DE, Wefers AK, Reinhardt A, Coras R, Ruf VC, Schmid S et al (2019) Rosette-forming glioneuronal tumors share a distinct DNA methylation profile and mutations in FGFR1, with recurrent co-mutation of PIK3CA and NF1. Acta Neuropathol 138:497-504. https://doi.org/10.1007/s00401-019-02038-4

34. Solomon DA, Wood MD, Tihan T, Bollen AW, Gupta N, Phillips JJ, Perry A (2016) Diffuse midline gliomas with histone H3-K27M mutation: a series of 47 cases assessing the spectrum of morphologic variation and associated genetic alterations. Brain Pathol 26:569-580. https://doi.org/10. 1111/bpa.12336

35. Takai K, Tanaka S, Sota T, Mukasa A, Komori T, Taniguchi M (2017) Spinal Cord Astrocytoma with Isocitrate Dehydrogenase 1 Gene Mutation.
World Neurosurg 108: 991 e913-991 e916 https://doi.org/10.1016/j. wneu.2017.08.142

36. Wang L, Ge J, Lan Y, Shi Y, Luo Y, Tan Y, Liang M, Deng S, Zhang X, Wang W et al (2020) Tumor mutational burden is associated with poor outcomes in diffuse glioma. BMC Cancer 20:213. https://doi.org/10.1186/ s12885-020-6658-1

37. Weller M, van den Bent M, Tonn JC, Stupp R, Preusser M, Cohen-JonathanMoyal E, Henriksson R, Le Rhun E, Balana C, Chinot O et al (2017) European Association for Neuro-Oncology (EANO) guideline on the diagnosis and treatment of adult astrocytic and oligodendroglial gliomas. Lancet Oncol 18:e315-e329. https://doi.org/10.1016/S1470-2045(17)30194-8

38. Williams EA, Miller JJ, Tummala SS, Penson T, lafrate AJ, Juratli TA, Cahill DP (2018) TERT promoter wild-type glioblastomas show distinct clinical features and frequent PI3K pathway mutations. Acta Neuropathol Commun 6:106. https://doi.org/10.1186/s40478-018-0613-2

39. Wu G, Broniscer A, McEachron TA, Lu C, Paugh BS, Becksfort J, Qu C, Ding L, Huether R, Parker M et al (2012) Somatic histone H3 alterations in pediatric diffuse intrinsic pontine gliomas and non-brainstem glioblastomas. Nat Genet 44:251-253. https://doi.org/10.1038/ng.1102

40. Xiao R, Abdullah KG, Miller JA, Lubelski D, Steinmetz MP, Shin JH, Krishnaney AA, Mroz TE, Benzel EC (2016) Molecular and clinical prognostic factors for favorable outcome following surgical resection of adult intramedullary spinal cord astrocytomas. Clin Neurol Neurosurg 144:82-87. https://doi.org/10.1016/j.clineuro.2016.03.009

41. Yanamadala V, Koffie RM, Shankar GM, Kumar JI, Buchlak QD, Puthenpura V, Frosch MP, Gudewicz TM, Borges LF, Shin JH (2016) Spinal cord glioblastoma: 25years of experience from a single institution. J Clin Neurosci 27:138-141. https://doi.org/10.1016/j.jocn.2015.11.011

42. Yao K, Duan Z, Wang Y, Zhang M, Fan T, Wu B, Qi X (2019) Detection of H3K27M mutation in cases of brain stem subependymoma. Hum Pathol 84:262-269. https://doi.org/10.1016/j.humpath.2018.10.011

43. Yi S, Choi S, Shin DA, Kim DS, Choi J, Ha Y, Kim KN, Suh CO, Chang JH, Kim $\mathrm{SH}$ et al (2019) Impact of H3.3 K27M mutation on prognosis and survival of grade IV spinal cord glioma on the basis of new 2016 World Health Organization classification of the central nervous system. Neurosurgery 84:1072-1081. https://doi.org/10.1093/neuros/nyy150

44. Zhang M, lyer RR, Azad TD, Wang Q, Garzon-Muvdi T, Wang J, Liu A, Burger P, Eberhart C, Rodriguez FJ et al (2019) Genomic landscape of intramedullary spinal cord gliomas. Sci Rep 9:18722. https://doi.org/10.1038/ s41598-019-54286-9

45. Zhang YW, Chai RC, Cao R, Jiang WJ, Liu WH, Xu YL, Yang J, Wang YZ, Jia WQ (2020) Clinicopathological characteristics and survival of spinal cord astrocytomas. Cancer Med 9:6996-7006. https://doi.org/10.1002/cam4. 3364

\section{Publisher's Note}

Springer Nature remains neutral with regard to jurisdictional claims in published maps and institutional affiliations.

$$
\begin{aligned}
& \text { Ready to submit your research? Choose BMC and benefit from: } \\
& \text { - fast, convenient online submission } \\
& \text { - thorough peer review by experienced researchers in your field } \\
& \text { - rapid publication on acceptance } \\
& \text { - support for research data, including large and complex data types } \\
& \text { - gold Open Access which fosters wider collaboration and increased citations } \\
& \text { - maximum visibility for your research: over } 100 \mathrm{M} \text { website views per year }
\end{aligned}
$$

At $\mathrm{BMC}$, research is always in progress.

Learn more biomedcentral.com/submissions 